\title{
Isometries of Half Supersymmetric Time-Like Solutions In Five Dimensions
}

\author{
Jan B. Gutowski ${ }^{1}$ and Wafic A. Sabra ${ }^{2}$ \\ ${ }^{1}$ DAMTP, Centre for Mathematical Sciences \\ University of Cambridge \\ Wilberforce Road, Cambridge, CB3 0WA, UK \\ Email: J.B.Gutowski@damtp.cam.ac.uk \\ ${ }^{2}$ Centre for Advanced Mathematical Sciences and Physics Department \\ American University of Beirut \\ Lebanon \\ Email:ws00@aub.edu.lb
}

\begin{abstract}
Spinorial geometry techniques have recently been used to classify all half supersymmetric solutions in gauged five dimensional supergravity with vector multiplets. In this paper we consider solutions for which at least one of the Killing spinors generates a timelike Killing vector. We obtain coordinate transformations which considerably simplify the solutions, and in a number of cases, we obtain explicitly some additional Killing vectors which were hidden in the original analysis.
\end{abstract}




\section{Introduction}

The classification of supersymmetric supergravity solutions is of interest in a number of contexts, such as the conjectured AdS/CFT correspondence, the microscopic analysis of black hole entropy, and a deeper understanding of string theory dualities. In particular, in five dimensions, considerable progress has been made in the classification and the analysis of solutions preserving various fractions of supersymmetry of $N=2$ gauged supergravity [1]-[11]. In these theories, the only solution which preserves all of the supersymmetries is $A d S_{5}$ with vanishing gauge field strengths and constant scalars. The Killing spinor equations when expressed in terms of Dirac spinors are linear over $\mathbb{C}$, implying that supersymmetric solutions preserve $2,4,6$ or 8 of the supersymmetries. However in the ungauged theory it was found that supersymmetric solutions can only preserve 4 or 8 of supersymmetries [12, 13]. The first supersymmetric solutions which were constructed [1] have naked singularities or naked closed time-like curves. Domain walls and magnetic strings were also constructed in [2]. Later, a more systematic approach, motivated by the results of Tod [14], was employed to construct and classify supersymmetric solutions. Quarter supersymmetric solutions of minimal gauged $N=2, D=5$ supergravity where classified in [3]. The first asymptotically $A d S_{5}$ solutions with no closed time loops or naked singularities were constructed for the minimal supergravity theory in [4]. Generalisations to solutions with null Killing spinors as well as to solutions with vector multiplets were later given in [5, 6].

Half supersymmetric solutions posses two Dirac Killing spinors thus enabling the construction of two Killing vectors as bilinears in the Killing spinors. These vectors could be either time-like or null and thus there exists three classes of solutions, depending on the nature of the Killing spinors and vectors considered. All of these solutions have been fully classified in [7, 8], using the spinorial geometry method. In this approach to the analysis of the Killing spinor equations, the Killing spinors are expressed in terms of differential forms [15, 16, 17], which are further simplified by making appropriately chosen gauge transformations. These techniques were originally developed to analyse more complicated supergravity theories in ten and eleven dimensions, see for example [18, 19, 20, 21]; it is also straightforward to use them to analyse lower dimensional theories as well. The remaining class of supersymmetric solutions, preserving $3 / 4$ of the supersymmetry, has also been considered recently in [9] where it was shown, again using spinorial geometry techniques, that these solutions are merely cosets of $A d S_{5}$.

It must be noted that supersymmetric solutions are not automatically solutions of the equations of motion. For the $1 / 4$ supersymmetric time-like solutions, one must solve the gauge equations and the Bianchi identities in addition to the Killing spinor equations. In the null case, however, one must additionally solve one of the components of the Einstein equations of motion. For the $1 / 2$ supersymmetric solutions, considered in our present work, it was found that supersymmetry together with Bianchi identities are sufficient to imply that all the Einstein, gauge and scalar equations of motion are all satisfied.

Our present work is concerned with half supersymmetric solutions of gauged $N=$ 
2, $D=5$ supergravity. We examine the time-like solutions of [7], and find coordinate transformations which simplify these solutions and make it possible to extract some hidden symmetries. Our work is organised as follows. In section two we present the basics of the theories we are studying and their half supersymmetric solutions which are presented in six classes. As three of these classes are already presented in [7] in the most explicit possible form, we focus on the other three classes and find coordinate transformations that simplify them. This simplification is presented in section 3 .

\section{Half Supersymmetric Timelike Solutions}

In this section, we present a summary of all half supersymmetric solutions of gauged $N=2, D=5$ supergravity, for which at least one of the Killing spinors generates a timelike Killing vector. Such solutions were completely classified in [7] and are specified in terms of a spacetime metric, together with a number of scalars $X^{I}$ and 2-form gauge field strengths $F^{I}$. In the classification of these solutions, the Killing spinors were expressed as differential forms on $\Lambda^{*}\left(\mathbb{R}^{2}\right) \otimes \mathbb{C}$. One starts with the most generic form for a symplectic Majorana spinor, then, by applying appropriately chosen gauge transformations, the spinor can be written in a number of particularly simple canonical forms. The conditions for quarter supersymmetric solutions with a time-like Killing vector (generated from the Killing spinor) are then derived. The conditions are then substituted into the Killing spinor equations, acting on the second spinor, and further constraints on the Kähler base are then determined. Using the integrability conditions of the Killing spinor equations, it was demonstrated that for a given background preserving at least half of the supersymmetry, where at least one of the Killing spinors generates a time-like Killing vector, all of the Einstein, gauge and scalar field equations of motion hold automatically provided that the Bianchi identity is satisfied.

In listing all possible solutions, it will be particularly useful to introduce coordinates $t, x$ and decompose the mostly negative signature metric as

$$
d s^{2}=f^{4}(d t+\Omega)^{2}-f^{-2} d s_{\mathbf{B}}^{2},
$$

where $\frac{\partial}{\partial t}$ is a Killing vector which is a symmetry of the whole solution, $d s_{\mathbf{B}}^{2}$ is the metric on a 4-manifold $\mathbf{B}$ which is constrained by supersymmetry to be Kähler; $f$ is a $t$-independent function and $\Omega$ is a $t$-independent 1 -form on $\mathbf{B}$. The bosonic action of the theory is

$S=\frac{1}{16 \pi G} \int\left(-R+2 \chi^{2} \mathcal{V}\right) * 1+Q_{I J}\left(d X^{I} \wedge \star d X^{J}-F^{I} \wedge * F^{J}\right)-\frac{C_{I J K}}{6} F^{I} \wedge F^{J} \wedge A^{K}$

where $I, J, K$ take values $1, \ldots, n$. The scalar fields $X^{I}$ are subject to the constraint

$$
X_{I} X^{I}=1
$$

where

$$
X_{I}=\frac{1}{6} C_{I J K} X^{J} X^{K}
$$


The coupling $Q_{I J}$ and the scalar potential depend on the scalars via

$$
\begin{aligned}
Q_{I J} & =\frac{9}{2} X_{I} X_{J}-\frac{1}{2} C_{I J K} X^{K} \\
\mathcal{V} & =9 V_{I} V_{J}\left(X^{I} X^{J}-\frac{1}{2} Q^{I J}\right)
\end{aligned}
$$

where $V_{I}$ are constants.

Bosonic backgrounds are said to be supersymmetric if there exists a spinor $\epsilon^{a}$ for which the supersymmetry variations of the gravitino and dilatino vanish in the given background. For the gravitino this requires

$$
\left[\nabla_{\mu}+\frac{1}{8} X_{I}\left(\gamma_{\mu} F_{\rho \sigma}^{I} \gamma^{\rho \sigma}-6 F_{\mu \rho}^{I} \gamma^{\rho}\right)\right] \epsilon^{a}-\frac{\chi}{2} V_{I}\left(X^{I} \gamma_{\mu}-3 A_{\mu}^{I}\right) \epsilon^{a b} \epsilon^{b}=0
$$

and for the dilatino it requires

$$
\left[\frac{1}{4}\left(Q_{I J} \gamma^{\mu \nu} F_{\mu \nu}^{J}+3 \gamma^{\mu} \nabla_{\mu} X_{I}\right) \epsilon^{a}-\frac{3 \chi}{2} V_{I} \epsilon^{a b} \epsilon^{b}\right] \partial_{r} X^{I}=0 .
$$

The Einstein equations derived from (2.2) are given by

$$
R_{\alpha \beta}+Q_{I J}\left(F_{\alpha \lambda}^{I} F_{\beta}^{J}{ }^{\lambda}-\nabla_{\alpha} X^{I} \nabla_{\beta} X^{J}-\frac{1}{6} g_{\alpha \beta} F_{\mu \nu}^{I} F^{J \mu \nu}\right)-\frac{2}{3} g_{\alpha \beta} \chi^{2} \mathcal{V}=0
$$

The Maxwell equations are

$$
d\left(Q_{I J} \star F^{J}\right)=-\frac{1}{4} C_{I J K} F^{J} \wedge F^{K}
$$

The scalar equations are

$$
\begin{aligned}
& {\left[-d\left(\star d X_{I}\right)+\left(X_{M} X^{P} C_{N P I}-\frac{1}{6} C_{M N I}\right)\left(F^{M} \wedge \star F^{N}-d X^{M} \wedge \star d X^{N}\right)\right.} \\
& \left.-\frac{3}{2} \chi^{2} V_{M} V_{N} Q^{M L} Q^{N P} C_{L P I} \mathrm{dvol}\right] \partial_{r} X^{I}=0 .
\end{aligned}
$$

We refer the reader to [7] for the details of the analysis of the Killing spinor equations. The solutions can be divided into six classes:

(1) The Kähler base metric has co-ordinates $\tau, \eta, u, v$ and is given by

$$
\begin{aligned}
d s_{\mathbf{B}}^{2} & =H^{2}\left(d \tau+\eta\left(\frac{\partial Y}{\partial u}+H^{2} \sin Y\right) d u+\eta \frac{\partial Y}{\partial v} d v\right)^{2}+\frac{d v^{2}}{H^{2}}+H^{2} v^{2} \sin ^{2} Y d u^{2} \\
& +H^{2}\left(d \eta-\eta \cot Y \frac{\partial Y}{\partial u} d u-\eta\left(\cot Y \frac{\partial Y}{\partial v}+\frac{1}{v}\right) d v\right)^{2}
\end{aligned}
$$


and

$$
\begin{aligned}
\Omega= & -\frac{1}{2 c v}\left(H^{2}+\frac{c^{2} v^{2}}{f^{6}}\right) d \tau+\frac{\eta}{c v}\left(\frac{1}{2} \theta \sin Y-H^{2} \frac{\partial Y}{\partial v}\right) d v \\
& -\eta\left(\frac{H^{2}}{c v}\left(\frac{\partial Y}{\partial u}+H^{2} \sin Y\right)+\frac{1}{2} \sin Y\left(\frac{\theta H^{2}}{c} \cos Y+\frac{c}{f^{6}} H^{2} v-\frac{1}{c v} H^{4}\right)\right) d u
\end{aligned}
$$

and the scalars $X^{I}$ and function $f$ are constrained by

$$
\begin{aligned}
\frac{\partial}{\partial u}\left(\frac{X_{I}}{f^{2}}\right) & =\frac{\chi H^{2} V_{I} \sin ^{2} Y}{c}, \\
\frac{\partial}{\partial v}\left(\frac{X_{I}}{f^{2}}\right) & =\frac{1}{v}\left(\frac{\chi V_{I}(\cos Y-1)}{c}-\frac{X_{I}}{f^{2}}\right) .
\end{aligned}
$$

For this solution, the scalars $X^{I}$ and $f$ depend only on $u, v ; c, \theta$ are constants with $c \neq 0$, and $H, Y$ are functions of $u, v$ such that $\sin Y \neq 0$, which are constrained by

$$
\begin{aligned}
\frac{\partial H^{2}}{\partial u} & =H^{2} v \sin ^{2} Y\left(3 \frac{\chi c v V_{I} X^{I}}{f^{4}}-\theta\right) \\
\frac{\partial H^{2}}{\partial v} & =-\frac{c v}{f^{4}}\left(3 \chi V_{I} X^{I}+\frac{c}{f^{2}}\right)+\cos Y\left(3 \frac{\chi c v V_{I} X^{I}}{f^{4}}-\theta\right),
\end{aligned}
$$

and

$$
\begin{aligned}
& \frac{\partial Y}{\partial u}=\sin Y\left(-H^{2}+3 \frac{\chi c v^{2} V_{I} X^{I}}{f^{4}}+\frac{c^{2} v^{2}}{f^{6}}\right)+\frac{v}{2} \sin 2 Y\left(3 \frac{\chi c v V_{I} X^{I}}{f^{4}}-\theta\right) \\
& \frac{\partial Y}{\partial v}=-\frac{1}{H^{2}} \sin Y\left(3 \frac{\chi c v V_{I} X^{I}}{f^{4}}-\theta\right)
\end{aligned}
$$

If $\theta \neq 0$, then one can integrate up the constraints (2.14) to obtain

$$
X_{I}=f^{2}\left(\frac{q_{I}}{v}+\frac{\chi}{c}\left(\frac{c^{2} v}{f^{6} \theta}-\frac{H^{2}}{\theta v}-1\right) V_{I}\right) .
$$

The gauge field strengths are given by

$$
\begin{aligned}
F^{I}= & d\left[f^{2} X^{I}(d t+\Omega)+\frac{c v X^{I}}{f^{4}}\left(d \tau+\eta H^{2} \sin Y d u\right)\right. \\
& \left.-\frac{3 \chi \eta}{f^{2}} \sin Y\left(X^{I} X^{J}-\frac{1}{2} Q^{I J}\right) V_{J}\left(-H^{2} v(1+\cos Y) d u+d v\right)\right] .
\end{aligned}
$$


(2) For the second class of solutions, one can choose a co-ordinate $v$ on $\mathbf{B}$ together with three $v$-independent 1 -forms $\sigma^{i}(i=1,2,3)$ on $\mathbf{B}$ orthogonal to $\frac{\partial}{\partial v}$. One also has constants $c, \theta(c \neq 0)$ and the solution takes one of three types according as $c \theta$ is negative, zero or positive. If $\theta \neq 0$ then

$$
d s_{\mathbf{B}}^{2}=\frac{1}{\theta v+c^{2} v^{2} f^{-6}} d v^{2}+\frac{v}{\theta^{2}}\left(\theta+c^{2} v f^{-6}\right)\left(\sigma^{1}\right)^{2}+\frac{v}{|\theta|}\left(\left(\sigma^{2}\right)^{2}+\left(\sigma^{3}\right)^{2}\right),
$$

and if $\theta=0$,

$$
d s_{\mathbf{B}}^{2}=\frac{1}{c^{2} v^{2} f^{-6}} d v^{2}+4 c^{8} f^{-6} v^{2}\left(\sigma^{1}\right)^{2}+2 c^{3} v\left(\left(\sigma^{2}\right)^{2}+\left(\sigma^{3}\right)^{2}\right) .
$$

The 1-forms $\sigma^{i}$ satisfy

$$
\begin{array}{lll}
d \sigma^{i}=-\frac{1}{2} \epsilon_{i j k} \sigma^{j} \wedge \sigma^{k} & : & \text { if } c \theta>0, \\
d \sigma^{1}=\sigma^{2} \wedge \sigma^{3}, d \sigma^{2}=\sigma^{1} \wedge \sigma^{3}, d \sigma^{3}=-\sigma^{1} \wedge \sigma^{2} & : & \text { if } c \theta<0, \\
d \sigma^{1}=\sigma^{2} \wedge \sigma^{3}, d \sigma^{2}=d \sigma^{3}=0 & : & \text { if } c \theta=0,
\end{array}
$$

If $\theta \neq 0$ then

$$
\Omega=-\frac{c v}{\theta} f^{-6} \sigma^{1}
$$

whereas if $\theta=0$ then

$$
\Omega=2 c^{4} v f^{-6} \sigma^{1}
$$

In all cases, the scalars $f$ and $X^{I}$ are constrained by

$$
X_{I}=\frac{f^{2}}{c}\left(-2 \chi V_{I}+\frac{\rho_{I}}{\sqrt{2} v}\right)
$$

for constants $\rho_{I}$ and

$$
F^{I}=d\left(f^{2} X^{I} d t\right)
$$

(3) For the third class of solutions one can again choose a co-ordinate $v$ on $\mathbf{B}$ together with three $v$-independent 1 -forms $\sigma^{i}(i=1,2,3)$ on $\mathbf{B}$, orthogonal to $\frac{\partial}{\partial v}$. For these solutions, the scalars $X^{I}$ are constant, and it is convenient to define

$$
\Lambda=c \theta+9 \sqrt{2} \chi^{2}\left(X^{I} X^{J}-\frac{1}{2} Q^{I J}\right) V_{I} V_{J}
$$

for constants $c, \theta(c \neq 0)$. The scalar $f$ is given by

$$
f^{2}=\sqrt{2} c v
$$

The solution takes one of three types. If $\Lambda \neq 0$ then

$$
\begin{aligned}
d s_{\mathbf{B}}^{2} & =\frac{1}{\left(\frac{1}{2 \sqrt{2} c v}-\theta v+\frac{3 \chi}{c} V_{I} X^{I}\right)} d v^{2}+\frac{c^{2}}{\Lambda^{2}}\left(\frac{1}{2 \sqrt{2} c v}-\theta v+\frac{3 \chi}{c} V_{I} X^{I}\right)\left(\sigma^{1}\right)^{2} \\
& +\frac{c v}{|\Lambda|}\left(\left(\sigma^{2}\right)^{2}+\left(\sigma^{3}\right)^{2}\right)
\end{aligned}
$$


and if $\Lambda=0$,

$$
\begin{aligned}
d s_{\mathbf{B}}^{2} & =\frac{1}{\left(\frac{1}{2 \sqrt{2} c v}-\theta v+\frac{3 \chi}{c} V_{I} X^{I}\right)} d v^{2}+2 c^{2}\left(\frac{1}{2 \sqrt{2} c v}-\theta v+\frac{3 \chi}{c} V_{I} X^{I}\right)\left(\sigma^{1}\right)^{2} \\
& +\sqrt{2} c v\left(\left(\sigma^{2}\right)^{2}+\left(\sigma^{3}\right)^{2}\right)
\end{aligned}
$$

The 1-forms $\sigma^{i}$ satisfy

$$
\begin{array}{lll}
d \sigma^{i}=-\frac{1}{2} \epsilon_{i j k} \sigma^{j} \wedge \sigma^{k} & : & \text { if } \Lambda>0 \\
d \sigma^{1}=\sigma^{2} \wedge \sigma^{3}, d \sigma^{2}=\sigma^{1} \wedge \sigma^{3}, d \sigma^{3}=-\sigma^{1} \wedge \sigma^{2} & : & \text { if } \Lambda<0 \\
d \sigma^{1}=\sigma^{2} \wedge \sigma^{3}, d \sigma^{2}=d \sigma^{3}=0 & : & \text { if } \Lambda=0
\end{array}
$$

If $\Lambda \neq 0$ then

$$
\begin{aligned}
\Omega & =\frac{1}{\Lambda c v^{2}}\left(\frac{1}{2 \sqrt{2}}+\frac{3 \chi v}{2} V_{I} X^{I}\right) \sigma^{1} \\
F^{I} & =d\left(\sqrt{2} c v X^{I} d t+\frac{3 \chi}{\sqrt{2} \Lambda}\left(Q^{I J}-X^{I} X^{J}\right) V_{J} \sigma^{1}\right)
\end{aligned}
$$

whereas if $\Lambda=0$, then

$$
\begin{aligned}
\Omega & =\frac{\sqrt{2}}{c v^{2}}\left(\frac{1}{2 \sqrt{2}}+\frac{3 \chi v}{2} V_{I} X^{I}\right) \sigma^{1} \\
F^{I} & =d\left(\sqrt{2} c v X^{I} d t+3 \chi\left(Q^{I J}-X^{I} X^{J}\right) V_{J} \sigma^{1}\right) .
\end{aligned}
$$

(4) For the fourth class of solution, the scalars $X^{I}$ are constant $\left(V_{I} X^{I} \neq 0\right)$, and

$$
f=1 \text {. }
$$

The Kähler base metric is the product of two 2-manifolds

$$
d s_{\mathbf{B}}^{2}=d s^{2}\left(M_{1}\right)+d s^{2}\left(M_{2}\right)
$$

where $M_{1}$ is $\mathbb{H}^{2}$ with Ricci scalar $R=-18 \chi^{2}\left(V_{I} X^{I}\right)^{2}$, and $M_{2}$ is $\mathbb{H}^{2}, \mathbb{R}^{2}$ or $\mathbb{S}^{2}$ with Ricci scalar $R=18 \chi^{2}\left(Q^{I J}-X^{I} X^{J}\right) V_{I} V_{J}$. In addition, we have

$$
d \Omega=3 \chi V_{I} X^{I} \mathrm{dvol}\left(M_{1}\right), \quad F^{I}=3 \chi\left(X^{I} X^{J}-Q^{I J}\right) V_{J} \mathrm{dvol}\left(M_{2}\right)
$$

where dvol $\left(M_{1}\right)$, dvol $\left(M_{2}\right)$ are the volume forms of $M_{1}, M_{2}$.

(5) For the fifth class of solution, one takes co-ordinates $\psi, \phi, x^{1}, x^{2}$ on the base space $\mathbf{B}$, whose metric is given by

$$
d s_{\mathbf{B}}^{2}=e^{\sqrt{2} \varrho^{2} \psi}\left[(d \phi+\beta)^{2}+d \psi^{2}+T^{2}\left(\left(d x^{1}\right)^{2}+\left(d x^{2}\right)^{2}\right)\right]
$$

where $\beta=\beta_{i}\left(x^{1}, x^{2}\right) d x^{i}, \varrho$ is a non-zero constant and $T=T\left(x^{1}, x^{2}\right)$ is a scalar. $\beta$ is constrained by the relation

$$
T^{2}=\frac{1}{\sqrt{2} \varrho^{2}}\left(\frac{\partial \beta_{2}}{\partial x^{1}}-\frac{\partial \beta_{1}}{\partial x^{2}}\right) .
$$


The scalars $X^{I}$ depend only on $x^{1}, x^{2}$, and

$$
f=e^{\frac{1}{\sqrt{2}} \varrho^{2} \psi} u
$$

for a function $u$ which depends only on $x^{1}, x^{2}$. There also exist two purely imaginary functions $\mathcal{G}, \mathcal{H}$ which depend only on $x^{1}, x^{2}$ and satisfy the constraints

$$
\frac{\partial}{\partial x^{2}}(T \mathcal{H})=\frac{\partial}{\partial x^{1}}(T \mathcal{G})
$$

and

$$
\frac{\partial}{\partial x^{1}}\left(\frac{\mathcal{H}}{T}\right)=\frac{\partial}{\partial x^{2}}\left(\frac{\mathcal{G}}{T}\right), \quad \frac{\partial}{\partial x^{1}}\left(\frac{\mathcal{G}}{T}\right)=-\frac{\partial}{\partial x^{2}}\left(\frac{\mathcal{H}}{T}\right) .
$$

The scalars $u$ and $X^{I}$ are constrained by

$$
X_{I}=u^{2} q_{I}+\chi u^{2}\left(-\frac{i}{\sqrt{2} \varrho^{3}}\left(\frac{1}{T} \frac{\partial \mathcal{G}}{\partial x^{2}}+\frac{\mathcal{H}}{T^{2}} \frac{\partial T}{\partial x^{1}}\right)+\frac{3 \chi}{\varrho^{4} u^{2}} V_{J} X^{J}\right) V_{I}
$$

for constant $q_{I}$, and $T$ satisfies the equation

$$
\square \log T+2 \varrho^{4} T^{2}=18 \chi^{2}\left(X^{I} X^{J}-\frac{1}{2} Q^{I J}\right) V_{I} V_{J} \frac{T^{2}}{u^{2}}
$$

where $\square=\left(\frac{\partial}{\partial x^{1}}\right)^{2}+\left(\frac{\partial}{\partial x^{2}}\right)^{2}$ is the Laplacian on $\mathbb{R}^{2}$. Finally, $\Omega$ is given by

$$
\begin{aligned}
\Omega & =-\frac{e^{-\sqrt{2} \varrho^{2} \psi}}{\sqrt{2}}\left[i T \varrho\left(-\mathcal{G} d x^{1}+\mathcal{H} d x^{2}\right)\right. \\
& \left.+\left(-\frac{i}{\sqrt{2}} \frac{1}{\varrho}\left(\frac{1}{T} \frac{\partial \mathcal{G}}{\partial x^{2}}+\frac{\mathcal{H}}{T^{2}} \frac{\partial T}{\partial x^{1}}\right)+\frac{3 \chi}{\varrho^{2} u^{2}} V_{I} X^{I}\right)(d \phi+\beta)\right]
\end{aligned}
$$

and the gauge field strengths are

$$
F^{I}=d\left(f^{2} X^{I}(d t+\Omega)\right)+6 \chi\left(X^{I} X^{J}-\frac{1}{2} Q^{I J}\right) V_{J} \frac{T^{2}}{u^{2}} d x^{1} \wedge d x^{2} .
$$

(6) For the sixth class of solutions, it is again convenient to introduce coordinates $\phi, \psi, x^{1}, x^{2}$ on the Kähler base. The base space metric is then

$$
d s_{\mathbf{B}}^{2}=d \phi^{2}+d \psi^{2}+T^{2}\left(\left(d x^{1}\right)^{2}+\left(d x^{2}\right)^{2}\right)
$$

where $T=T\left(x^{1}, x^{2}\right)$. The scalars $f$ and $X^{I}$ depend only on $x^{1}, x^{2}$. Again, there also exist two purely imaginary functions $\mathcal{G}, \mathcal{H}$ which depend only on $x^{1}, x^{2}$ and satisfy the constraints

$$
\frac{\partial}{\partial x^{2}}(T \mathcal{H})=\frac{\partial}{\partial x^{1}}(T \mathcal{G})
$$

and

$$
\frac{\partial}{\partial x^{1}}\left(\frac{\mathcal{H}}{T}\right)=\frac{\partial}{\partial x^{2}}\left(\frac{\mathcal{G}}{T}\right), \quad \frac{\partial}{\partial x^{1}}\left(\frac{\mathcal{G}}{T}\right)=-\frac{\partial}{\partial x^{2}}\left(\frac{\mathcal{H}}{T}\right)
$$


The scalars $f$ and $X^{I}$ are constrained via

$$
\begin{aligned}
\frac{\partial}{\partial x^{1}}\left(\frac{X_{I}}{f^{2}}\right) & =\sqrt{2} i \chi T \mathcal{H} V_{I} \\
\frac{\partial}{\partial x^{2}}\left(\frac{X_{I}}{f^{2}}\right) & =\sqrt{2} i \chi T \mathcal{G} V_{I}
\end{aligned}
$$

and $T$ satisfies

$$
\square \log T=18 \frac{\chi^{2}}{f^{2}}\left(X^{I} X^{J}-\frac{1}{2} Q^{I J}\right) V_{I} V_{J} T^{2}
$$

Finally, $\Omega$ is constrained by

$$
\begin{aligned}
d \Omega & =-\frac{i}{\sqrt{2}}\left(T \frac{\partial \mathcal{G}}{\partial x^{2}}+\mathcal{H} \frac{\partial T}{\partial x^{1}}\right)\left(d x^{1} \wedge d x^{2}-\frac{1}{T^{2}} d \phi \wedge d \psi\right) \\
& -\frac{3 \chi}{f^{4}} V_{I} X^{I}\left(T^{2} d x^{1} \wedge d x^{2}+d \phi \wedge d \psi\right),
\end{aligned}
$$

and the gauge field strengths are then given by

$$
F^{I}=d\left(f^{2} X^{I}(d t+\Omega)\right)+6 \chi \frac{T^{2}}{f^{2}} V_{J}\left(X^{I} X^{J}-\frac{1}{2} Q^{I J}\right) d x^{1} \wedge d x^{2}
$$

\section{Simplification of the Solutions}

The solutions of type (2), (3), (4) are given in the most explicit possible form. Hence, we shall concentrate on the solutions of type (1), (5), (6).

\subsection{Simplification of type (1) Solutions}

We find it convenient to simply the expression for the metric and gauge field strengths of these solutions by changing co-ordinates from $(t, \tau, \eta, u, v)$ to $\left(t^{\prime}, \phi, w, u, v\right)$. There are two cases, corresponding to $\theta \neq 0$ and $\theta=0$.

\subsubsection{Solutions with $\theta \neq 0$}

If $\theta \neq 0$, it is convenient to make the co-ordinate transformation

$$
\begin{aligned}
t & =t^{\prime}-\frac{w}{2 c}\left(H^{2}-c^{2} v^{2} f^{-6}\right) \cos Y-\frac{w v \theta}{2 c} \\
\eta & =v \sin Y w \\
\tau & =\phi+\frac{w}{\theta}\left(H^{2}-c^{2} v^{2} f^{-6}\right)+v w \cos Y
\end{aligned}
$$


In these new co-ordinates, the solution is specified by:

$$
\begin{aligned}
d s_{\mathbf{B}}^{2} & =H^{2}\left(d \phi+\left(v \cos Y+\theta^{-1}\left(H^{2}-c^{2} v^{2} f^{-6}\right)\right) d w\right)^{2} \\
& +H^{-2} d v^{2}+H^{2} v^{2} \sin ^{2} Y\left(d w^{2}+d u^{2}\right) \\
d t+\Omega & =d t^{\prime}-\frac{1}{2 c v}\left(H^{2}+c^{2} v^{2} f^{-6}\right) d \phi \\
& -\left(\frac{1}{2 c \theta v}\left(H^{4}-c^{4} v^{4} f^{-12}\right)+\frac{1}{c}\left(H^{2} \cos Y+\frac{\theta v}{2}\right)\right) d w \\
F^{I} & =d\left(f ^ { 2 } X ^ { I } \left(d t^{\prime}-\frac{1}{2 c v}\left(H^{2}-c^{2} v^{2} f^{-6}\right) d \phi\right.\right. \\
& \left.\left.-\left(\frac{1}{2 c v \theta}\left(H^{2}-c^{2} v^{2} f^{-6}\right)^{2}+\frac{H^{2}}{c} \cos Y+\frac{\theta v}{2 c}+c v^{2} f^{-6}\right) d w\right)\right) \\
X_{I} & =f^{2}\left(\frac{q_{I}}{v}+\frac{\chi}{c}\left(\frac{c^{2} v}{f^{6} \theta}-\frac{H^{2}}{\theta v}-1\right) V_{I}\right) .
\end{aligned}
$$

for constants $q_{I}$, where $Y, H$ are functions of $u, v(\sin Y \neq 0)$ satisfying the constraints (2.15) and (2.16). Note that $\frac{\partial}{\partial t^{\prime}}, \frac{\partial}{\partial \phi}$ and $\frac{\partial}{\partial w}$ are commuting Killing vectors which are also symmetries of the full solution.

\subsubsection{Solutions with $\theta=0$}

In the special case when $\theta=0$, note that

$$
d\left(\frac{c}{\chi} v f^{-2} X_{I}+v V_{I}\right)=V_{I}\left(\cos Y d v+H^{2} v \sin ^{2} Y d u\right)
$$

as not all of the $V_{I}$ vanish, fix some $\tilde{I}$ with $V_{\tilde{I}} \neq 0$, so that

$$
d\left(\frac{c}{\chi} v f^{-2} \frac{X_{\tilde{I}}}{V_{\tilde{I}}}+v\right)=\cos Y d v+H^{2} v \sin ^{2} Y d u
$$

It is also convenient to define

$$
X=v f^{-2} \frac{X_{\tilde{I}}}{V_{\tilde{I}}}
$$

The co-ordinate transformation is then given by

$$
\begin{aligned}
t & =t^{\prime}-\frac{w}{2 c}\left(H^{2}-c^{2} v^{2} f^{-6}\right) \cos Y \\
\eta & =v \sin Y w \\
\tau & =\phi-w\left(v+\frac{c}{\chi} X\right)+v w \cos Y .
\end{aligned}
$$


In these new co-ordinates, the solution is specified by

$$
\begin{aligned}
d s_{\mathbf{B}}^{2} & =H^{2}\left(d \phi+\left(v(\cos Y-1)-\frac{c}{\chi} X\right) d w\right)^{2} \\
& +H^{-2} d v^{2}+H^{2} v^{2} \sin ^{2} Y\left(d w^{2}+d u^{2}\right) \\
d t+\Omega & =d t^{\prime}-\frac{1}{2 c v}\left(H^{2}+c^{2} v^{2} f^{-6}\right) d \phi \\
& +\left(\frac{1}{2 c v}\left(H^{2}+c^{2} v^{2} f^{-6}\right)\left(v+\frac{c}{\chi} X\right)-\frac{H^{2}}{c} \cos Y\right) d w \\
F^{I} & =d\left(f ^ { 2 } X ^ { I } \left(d t^{\prime}-\frac{1}{2 c v}\left(H^{2}-c^{2} v^{2} f^{-6}\right) d \phi\right.\right. \\
& \left.\left.+\left(\frac{1}{2 c v}\left(H^{2}-c^{2} v^{2} f^{-6}\right)\left(v+\frac{c}{\chi} X\right)-\frac{H^{2}}{c} \cos Y-c v^{2} f^{-6}\right) d w\right)\right), \\
v f^{-2} X_{I} & =X V_{I}+q_{I},
\end{aligned}
$$

for constants $c, q_{I}\left(q_{\tilde{I}}=0\right)$, where $Y, H$ are functions of $u, v(\sin Y \neq 0)$ satisfying the constraints (2.15) and (2.16) with $\theta=0$.

Again, note that $\frac{\partial}{\partial t^{\prime}}, \frac{\partial}{\partial \phi}$ and $\frac{\partial}{\partial w}$ are commuting Killing vectors which are also symmetries of the full solution.

\subsection{Simplification of type (5) Solutions}

To simplify the solutions further, define

$$
Q=-\frac{i}{\sqrt{2}} \varrho\left(T^{-1} \frac{\partial \mathcal{G}}{\partial x^{2}}+\mathcal{H} T^{-2} \frac{\partial T}{\partial x^{1}}\right)+3 \chi u^{-2} V_{I} X^{I}
$$

so that

$$
\Omega=-\frac{e^{-\sqrt{2} \varrho^{2} \psi}}{\sqrt{2} \varrho^{2}}\left[i T \varrho^{3}\left(-\mathcal{G} d x^{1}+\mathcal{H} d x^{2}\right)+Q(d \phi+\beta)\right]
$$

and noting that

$$
d Q=\sqrt{2} i \varrho^{5}\left(T \mathcal{H} d x^{1}+T \mathcal{G} d x^{2}\right)
$$

one finds

$$
d\left[Q^{2}-\varrho^{6}\left(\frac{2 u^{-6}}{\varrho^{2}}+\mathcal{G}^{2}+\mathcal{H}^{2}\right)\right]=0
$$

and hence

$$
Q^{2}=\xi+\varrho^{6}\left(\frac{2 u^{-6}}{\varrho^{2}}+\mathcal{G}^{2}+\mathcal{H}^{2}\right)
$$

for constant $\xi$. Also note that

$$
u^{-2} X_{I}=\frac{\chi}{\varrho^{4}} Q V_{I}+q_{I}
$$

There are then a number of subclasses of solutions, according as to whether $\mathcal{H}^{2}+\mathcal{G}^{2} \neq$ 0 or $\mathcal{H}=\mathcal{G}=0$. 


\subsubsection{Solutions with $\mathcal{H}^{2}+\mathcal{G}^{2} \neq 0$}

Suppose we consider a neighbourhood in which $\mathcal{H}^{2}+\mathcal{G}^{2} \neq 0$. Note that as $T^{-1}(\mathcal{H}+i \mathcal{G})$ is a holomorphic function of $x^{1}+i x^{2}$, it follows that $\frac{T}{\mathcal{H}+i \mathcal{G}}$ is also a holomorphic function of $x^{1}+i x^{2}$, and so

$$
\frac{\partial}{\partial x^{1}}\left(\frac{T \mathcal{H}}{\mathcal{H}^{2}+\mathcal{G}^{2}}\right)=-\frac{\partial}{\partial x^{2}}\left(\frac{T \mathcal{G}}{\mathcal{H}^{2}+\mathcal{G}^{2}}\right), \quad \frac{\partial}{\partial x^{1}}\left(\frac{T \mathcal{G}}{\mathcal{H}^{2}+\mathcal{G}^{2}}\right)=\frac{\partial}{\partial x^{2}}\left(\frac{T \mathcal{H}}{\mathcal{H}^{2}+\mathcal{G}^{2}}\right)
$$

or equivalently

$$
\begin{aligned}
& d\left(\frac{T \mathcal{G}}{\mathcal{H}^{2}+\mathcal{G}^{2}} d x^{1}-\frac{T \mathcal{H}}{\mathcal{H}^{2}+\mathcal{G}^{2}} d x^{2}\right)=0, \\
& d\left(\frac{T \mathcal{H}}{\mathcal{H}^{2}+\mathcal{G}^{2}} d x^{1}+\frac{T \mathcal{G}}{\mathcal{H}^{2}+\mathcal{G}^{2}} d x^{2}\right)=0,
\end{aligned}
$$

hence one obtains (locally) real functions $z=z\left(x^{1}, x^{2}\right), y=y\left(x^{1}, x^{2}\right)$ such that

$$
\begin{aligned}
& \frac{T \mathcal{G}}{\mathcal{H}^{2}+\mathcal{G}^{2}} d x^{1}-\frac{T \mathcal{H}}{\mathcal{H}^{2}+\mathcal{G}^{2}} d x^{2}=i d z, \\
& \frac{T \mathcal{H}}{\mathcal{H}^{2}+\mathcal{G}^{2}} d x^{1}+\frac{T \mathcal{G}}{\mathcal{H}^{2}+\mathcal{G}^{2}} d x^{2}=i d y,
\end{aligned}
$$

with

$$
\begin{aligned}
\frac{\partial}{\partial z} & =\frac{i}{T}\left(\mathcal{G} \frac{\partial}{\partial x^{1}}-\mathcal{H} \frac{\partial}{\partial x^{2}}\right), \\
\frac{\partial}{\partial y} & =\frac{i}{T}\left(\mathcal{H} \frac{\partial}{\partial x^{1}}+\mathcal{G} \frac{\partial}{\partial x^{2}}\right) .
\end{aligned}
$$

Next, note that one can solve for the 1 -form $\beta$ to find (up to a total derivative which can be neglected)

$$
\beta=-\frac{i}{\varrho^{3}} \frac{Q T}{\mathcal{H}^{2}+\mathcal{G}^{2}}\left(-\mathcal{G} d x^{1}+\mathcal{H} d x^{2}\right)=-\frac{Q}{\varrho^{3}} d z
$$

and the expression for $\Omega$ can be further simplified to

$$
\Omega=-\frac{1}{\sqrt{2} \varrho^{2}} e^{-\sqrt{2} \varrho^{2} \psi}\left(Q d \phi-\frac{1}{\varrho^{3}}\left(\xi+2 \varrho^{2} u^{-6}\right) d z\right) .
$$

It is then straightforward to see that $\frac{\partial}{\partial z}$ is an additional Killing vector, which is also a symmetry of the full solution: $Q, X^{I}, u$ are functions only of $y$, and the metric on the Kähler base space simplifies to

$$
d s_{\mathbf{B}}^{2}=e^{\sqrt{2} \varrho^{2} \psi}\left(\left(d \phi-\frac{Q}{\varrho^{3}} d z\right)^{2}+d \psi^{2}-\left(\mathcal{H}^{2}+\mathcal{G}^{2}\right)\left(d z^{2}+d y^{2}\right)\right) .
$$


Finally, it is most useful to change co-ordinates from $(t, \psi, \phi, z, y)$ to $(t, \psi, \phi, z, Q)$; where

$$
d Q=-\sqrt{2} \varrho^{5}\left(\mathcal{H}^{2}+\mathcal{G}^{2}\right) d y .
$$

In these new co-ordinates, the solution is given by

$$
\begin{aligned}
d s_{\mathbf{B}}^{2} & =e^{\sqrt{2} \varrho \psi}\left[\left(d \phi-\frac{Q}{\varrho^{3}} d z\right)^{2}+d \psi^{2}+\left(2 u^{-6} \varrho^{-2}-\varrho^{-6}\left(Q^{2}-\xi\right)\right) d z^{2}\right. \\
& \left.+\frac{1}{2\left(2 u^{-6} \varrho^{8}-\varrho^{4}\left(Q^{2}-\xi\right)\right)} d Q^{2}\right] \\
u^{-2} X_{I} & =\frac{\chi}{\varrho^{4}} Q V_{I}+q_{I} \\
\Omega & =-\frac{1}{\sqrt{2} \varrho^{2}} e^{-\sqrt{2} \varrho^{2} \psi}\left(Q d \phi-\frac{1}{\varrho^{3}}\left(\xi+2 \varrho^{2} u^{-6}\right) d z\right), \\
F^{I} & =d\left(u^{2} e^{\sqrt{2} \varrho^{2} \psi} X^{I}(d t+\Omega)\right)+3 \sqrt{2} \chi \varrho^{-5} u^{-2} V_{I}\left(X^{I} X^{J}-\frac{1}{2} Q^{I J}\right) d z \wedge d Q \\
f & =e^{\frac{\varrho^{2}}{\sqrt{2}} \psi} u .
\end{aligned}
$$

for constants $\varrho \neq 0, q_{I}, \xi$. Note that $\frac{\partial}{\partial t}, \frac{\partial}{\partial \phi}, \frac{\partial}{\partial z}$ are commuting Killing vectors which are symmetries of the full solution.

\subsubsection{Solutions with $\mathcal{H}=\mathcal{G}=0$}

If $\mathcal{H}=\mathcal{G}=0$ then the scalars $X^{I}$ are constant, as is $u$. Without loss of generality, set $u=1$. With these constraints, the function $T$ which is introduced [7] must satisfy

$$
T^{-2} \square \log T=\Lambda
$$

where

$$
\Lambda=-2 \varrho^{4}+18 \chi^{2}\left(X^{I} X^{J}-\frac{1}{2} Q^{I J}\right) V_{I} V_{J}
$$

is constant. Let $M$ be a 2-manifold equipped with metric

$$
d s^{2}(M)=T^{2}\left(\left(d x^{1}\right)^{2}+\left(d x^{2}\right)^{2}\right) .
$$

Then (3.23) implies that $M$ has Ricci scalar ${ }^{(M)} R=-2 \Lambda$, so is isometric to $\mathbb{S}^{2}, \mathbb{R}^{2}$ or $\mathbb{H}^{2}$ according as $\Lambda<0, \Lambda=0$ or $\Lambda>0$ respectively. Note also that the 1 -form $\beta$ must satisfy

$$
d \beta=\sqrt{2} \varrho^{2} \mathrm{dvol}(M) .
$$


Hence, to summarize, these solutions have constant $X^{I}$, and

$$
\begin{aligned}
d s_{\mathbf{B}}^{2} & =e^{\sqrt{2} \varrho^{2} \psi}\left((d \phi+\beta)^{2}+d \psi^{2}+d s^{2}(M)\right), \quad d \beta=\sqrt{2} \varrho^{2} \mathrm{dvol}(M), \\
{ }^{(M)} R & =4 \varrho^{4}-36 \chi^{2}\left(X^{I} X^{J}-\frac{1}{2} Q^{I J}\right) V_{I} V_{J}, \\
\Omega & =-3 \chi V_{I} X^{I} \frac{e^{-\sqrt{2} \varrho^{2} \psi}}{\sqrt{2} \varrho^{2}}(d \phi+\beta), \\
F^{I} & =d\left(e^{\sqrt{2} \varrho^{2} \psi} X^{I}(d t+\Omega)\right)+6 \chi\left(X^{I} X^{J}-\frac{1}{2} Q^{I J}\right) V_{J} \mathrm{dvol}(M), \\
f & =e^{\frac{\varrho^{2}}{\sqrt{2}} \psi}
\end{aligned}
$$

where $\varrho$ is a non-zero constant.

Note that $\frac{\partial}{\partial t}$ and $\frac{\partial}{\partial \phi}$ are commuting Killing vectors which are symmetries of the full solution. A Killing vector of $M$ can also be obtained which commutes with both

$\frac{\partial}{\partial t}$ and $\frac{\partial}{\partial \phi}$ and is also a symmetry of the full solution. So again, there are three commuting Killing vectors which are symmetries of the full solution.

\subsection{Simplification of type (6) Solutions}

Again, for these solutions, there are two sub-classes, according as $\mathcal{G}^{2}+\mathcal{H}^{2} \neq 0$, or $\mathcal{G}=\mathcal{H}=0$.

\subsubsection{Solutions with $\mathcal{H}^{2}+\mathcal{G}^{2} \neq 0$}

In order to simplify the solutions of type (6), note that the functions $\mathcal{G}, \mathcal{H}, T$ satisfy the same constraints (2.42) and (2.43) as the type (5) solutions, and hence we again introduce co-ordinates $z, y$ such that

$$
\begin{aligned}
\frac{T \mathcal{G}}{\mathcal{H}^{2}+\mathcal{G}^{2}} d x^{1}-\frac{T \mathcal{H}}{\mathcal{H}^{2}+\mathcal{G}^{2}} d x^{2} & =i d z \\
\frac{T \mathcal{H}}{\mathcal{H}^{2}+\mathcal{G}^{2}} d x^{1}+\frac{T \mathcal{G}}{\mathcal{H}^{2}+\mathcal{G}^{2}} d x^{2} & =i d y
\end{aligned}
$$

with

$$
\begin{aligned}
\frac{\partial}{\partial z} & =\frac{i}{T}\left(\mathcal{G} \frac{\partial}{\partial x^{1}}-\mathcal{H} \frac{\partial}{\partial x^{2}}\right) \\
\frac{\partial}{\partial y} & =\frac{i}{T}\left(\mathcal{H} \frac{\partial}{\partial x^{1}}+\mathcal{G} \frac{\partial}{\partial x^{2}}\right) .
\end{aligned}
$$

It is also convenient to define the scalar $Q$ by

$$
Q^{2}=-\left(\mathcal{G}^{2}+\mathcal{H}^{2}\right)
$$


Then the constraints (2.42) and (2.43) imply that $Q, f$ and $X^{I}$ are functions only of $y$, with

$$
\frac{d}{d y}\left(f^{-2} X_{I}\right)=\sqrt{2} \chi Q^{2} V_{I}
$$

Next, note that

$$
\begin{aligned}
d \Omega & =\left(\frac{1}{\sqrt{2}} \frac{d \log Q}{d y}-3 \chi f^{-4} V_{I} X^{I}\right) d \phi \wedge d \psi \\
& +\left(-\frac{1}{\sqrt{2}} \frac{d \log Q}{d y}-3 \chi f^{-4} V_{I} X^{I}\right) T^{2} d x^{1} \wedge d x^{2}
\end{aligned}
$$

The integrability condition of this constraint is given by

$$
\frac{1}{\sqrt{2}} \frac{d \log Q}{d y}-3 \chi f^{-4} V_{I} X^{I}=\xi
$$

for constant $\xi$.

It is then straightforward to show that

$$
d \Omega=\xi d \phi \wedge d \psi+d\left(\frac{1}{\sqrt{2}}\left(\frac{1}{2} Q^{2}+f^{-6}\right) d z\right)
$$

and also

$$
F^{I}=d\left(f^{2} X^{I}(d t+\Omega)-\sqrt{2} f^{-4} X^{I} d z\right) .
$$

Finally, consider the constraints (3.30) and (3.32). As not all $V_{I}$ vanish, choose $\tilde{I}$ such that $V_{\tilde{I}} \neq 0$, then (3.30) implies

$$
Q^{2}=\frac{1}{\sqrt{2} \chi} \frac{d}{d y}\left(f^{-2} \frac{X_{\tilde{I}}}{V_{\tilde{I}}}\right) .
$$

It is then convenient to define

$$
X=f^{-2} \frac{X_{\tilde{I}}}{V_{\tilde{I}}} .
$$

Combining all of the above constraints, one finds that the solution is specified by

$$
\begin{aligned}
f^{-2} X_{I} & =X V_{I}+q_{I}, \quad \text { where } X \text { satisfies } \frac{1}{4 \chi} \frac{d X}{d y}-\frac{1}{2} f^{-6}=\frac{\xi}{\sqrt{2} \chi} X \\
d s_{\mathbf{B}}^{2} & =d \phi^{2}+d \psi^{2}+2 \sqrt{2}\left(\frac{1}{2} f^{-6}+\frac{\xi}{\sqrt{2} \chi} X\right)\left(d y^{2}+d z^{2}\right), \\
d \Omega & =\xi d \phi \wedge d \psi+d\left(\left(\sqrt{2} f^{-6}+\frac{\xi}{\sqrt{2} \chi} X\right) d z\right), \\
F^{I} & =d\left(f^{2} X^{I}(d t+\Omega)-\sqrt{2} f^{-4} X^{I} d z\right) .
\end{aligned}
$$

for constants $q_{I}\left(q_{\tilde{I}}=0\right), \xi$.

Note that $\frac{\partial}{\partial t}, \frac{\partial}{\partial \phi}, \frac{\partial}{\partial z}$ are commuting Killing vectors which are symmetries of the full solution. 


\subsubsection{Solutions with $\mathcal{H}=\mathcal{G}=0$}

For these solutions, the scalars $X^{I}$ and $f$ are constant; without loss of generality set

$$
f=1 .
$$

Then

$$
d s_{B}^{2}=d \phi^{2}+d \psi^{2}+d s^{2}(M)
$$

where $M$ is a 2-manifold which is either $\mathbb{S}^{2}, \mathbb{R}^{2}$ or $\mathbb{H}^{2}$ according as the Ricci scalar

$$
{ }^{(M)} R=-36 \chi^{2}\left(X^{I} X^{J}-\frac{1}{2} Q^{I J}\right) V_{I} V_{J}
$$

is positive, zero, or negative. In addition, one has

$$
\begin{aligned}
d \Omega & =-3 \chi V_{I} X^{I}(\operatorname{dvol}(M)+d \phi \wedge d \psi) \\
F^{I} & =-3 \chi X^{I} X^{J} V_{J} d \phi \wedge d \psi+3 \chi\left(X^{I} X^{J}-Q^{I J}\right) V_{J} \operatorname{dvol}(M) .
\end{aligned}
$$

It is clear that this solution also admits three Killing vectors which are symmetries of the full solution.

\section{Summary}

In summary we have revisited the classification of $1 / 2$ supersymmetric solutions of the theory of $N=2, D=5$ supergravity which have at least one time-like Killing spinor. Three of the six classes of these solutions were given in their most explicit form in [7]. Our purpose was to recast the remaining three solutions in a form which enabled us to extract some hidden isometries of the metric solutions. We found coordinate transformations that simplified these three classes of solutions and allowed the explicit construction of their Killing vectors. It is of interest to investigate whether there are any regular asymptotically $A d S_{5}$ black ring solutions. Supersymmetric rings exist in the ungauged theory [23, 24, 25, 26] and it is known that supersymmetry is fully restored at the ring horizon. Furthermore, supersymmetric regular asymptotically $A d S_{5}$ black holes undergo supersymmetry enhancement from $1 / 4$ to $1 / 2$ supersymmetry in their near horizon limits. So, if $A d S_{5}$ black rings exist in the gauged theory, one might also expect that supersymmetry is enhanced from $1 / 4$ to $1 / 2$ at the horizon.

Recent work [22] has shown that there are no black rings which have horizons with a $[U(1)]^{2}$ symmetry. In our work, we have shown that the $1 / 2$ supersymmetric solutions in the "timelike" class possess, in addition to the timelike Killing vector generated by the timelike Killing spinor, two further commuting Killing vectors. However, these two additional Killing vectors are not necessarily spacelike. Hence, in order to determine if there exists a $1 / 2$ supersymmetric solution corresponding to the nearhorizon geometry of a black ring, it will be necessary to construct a more detailed analysis of half-supersymmetric near horizon geometries. The simplifications to the half-supersymmetric solutions presented in this work will be useful in constructing such a classification. 


\section{Acknowledgments}

The work of W. A. Sabra was supported in part by the National Science Foundation under grant number PHY-0703017.

\section{References}

[1] K. Behrndt, A. H. Chamseddine and W. A. Sabra, BPS Black Holes in $N=2$ Five-dimensional AdS Supergravity, Phys. Lett. B442 (1998) 97; |hep-th/9807187|, D. Klemm and W. A. Sabra, Charged Rotating Black Holes in 5d Einstein-Maxwell-(A)dS Gravity, Phys. Lett. B503 (2001) 147; |hep-th/0010200|, General (anti-)de Sitter Black Holes in Five Dimensions, JHEP 02 (2001) 031; [hep-th/0011016.

[2] S. L. Cacciatori, D. Klemm and W. A. Sabra, Supersymmetric Domain Walls and Strings in D=5 Gauged Supergravity Coupled to Vector Multiplets, JHEP 03 (2003) 023; hep-th/0302218], D. Klemm and W. A. Sabra, Supersymmetry of Black Strings in D=5 Gauged Supergravities, Phys. Rev. D62 (2000) 024003; hep-th/0001131.

[3] J. P. Gauntlett and J. B. Gutowski, All Supersymmetric Solutions of Minimal Gauged Supergravity in Five Dimensions, Phys. Rev. D68 (2003) 105009; hep-th/0304064.

[4] J. B. Gutowski and H. S. Reall, Supersymmetric AdS $S_{5}$ Black Holes, JHEP 02 (2004) 006; [hep-th/0401042].

[5] J. B. Gutowski and H. S. Reall, General Supersymmetric AdS $S_{5}$ Black Holes, JHEP 04 (2004) 048; hep-th/0401129].

[6] J. B. Gutowski and W. A. Sabra, General Supersymmetric Solutions of FiveDimensional Supergravity, JHEP 10 (2005) 039; [hep-th/0505185].

[7] J. B. Gutowski and W. A. Sabra, Half-Supersymmetric Solutions in FiveDimensional Supergravity; JHEP 12 (2007) 025; arXiv:0706.3147].

[8] J. Grover, J. B. Gutowski and W. A. Sabra, Null Half-Supersymmetric Solutions in Five-Dimensional Supergravity; arXiv:0802.0231.

[9] J. Grover, J. B. Gutowski and W. A. Sabra, Vanishing Preons in the Fifth Dimension, Class. Quant. Grav. 24 (2007) 417; [hep-th/0608187], J. FigueroaO'Farrill, J. B. Gutowski and W. A. Sabra, The Return of the Four-and Fivedimensional Preons, Class. Quantum Grav. 24 (2007) 4429; arXiv:0705.2778.

[10] H. K. Kunduri, J. Lucietti, Near-horizon geometries of supersymmetric AdS(5) black holes, JHEP 12 (2007) 015; arXiv:0708.3695]. 
[11] Hari K. Kunduri, James Lucietti and Harvey S. Reall, Supersymmetric multicharge $A d S_{5}$ black holes, JHEP 04 (2006) 036; hep-th/0601156].

[12] J. P. Gauntlett, J. B. Gutowski, C. M. Hull, S. Pakis, H. S. Reall, All supersymmetric solutions of minimal supergravity in five dimensions, Class. Quant. Grav. 20 (2003) 4587; [hep-th/0209114].

[13] J. B. Gutowski, Uniqueness of Five-Dimensional Supersymmetric Black Holes, JHEP 08 (2004) 049; hep-th/0404079.

[14] K. P. Tod, All Metrics Admitting Supercovariantly Constant Spinors, Phys. Lett. B121 (1983) 241.

[15] H. Blaine Lawson and Marie-Louise Michelsohn, Spin Geometry, Princeton University Press (1989).

[16] McKenzie Y. Wang, Parallel Spinors and Parallel Forms, Ann. Global Anal Geom. 7, No 1 (1989), 59.

[17] F. R. Harvey, Spinors and Calibrations, Academic Press, London (1990).

[18] J. Gillard, U. Gran and G. Papadopoulos, The Spinorial Geometry of Supersymmetric Backgrounds, Class. Quant. Grav. 22 (2005) 1033; hep-th/0410155.

[19] U. Gran, J. B. Gutowski and G. Papadopoulos, The Spinorial Geometry of Supersymmetric IIB Backgrounds, Class. Quant. Grav. 22 (2005) 2453; hep-th/0501177].

[20] U. Gran, J. B. Gutowski, G. Papadopoulos and D. Roest, Maximally Supersymmetric G-Backgrounds of IIB Supergravity, Nucl. Phys. B753 (2006) 118; hep-th/0604079.

[21] U. Gran, J. B. Gutowski, G. Papadopoulos and D. Roest, N=31 is not IIB, JHEP 02 (2007) 044; hep-th/0606049.

[22] H. K. Kunduri, J. Lucietti and H. S. Reall, Do Supersymmetric Anti-de Sitter Black Rings Exist? JHEP 02 (2007) 026; hep-th/0611351.

[23] H. Elvang, R. Emparan, D. Mateos and H. S. Reall, A Supersymmetric Black Ring, Phys. Rev. Lett. 93 (2004) 211302; hep-th/0407065.

[24] H. Elvang, R. Emparan, D. Mateos and H. S. Reall, Supersymmetric Black Rings and Three-charge Supertubes, Phys. Rev. D71 (2005) 024033; hep-th/0408120].

[25] J. P. Gauntlett and J. B. Gutowski, Concentric Black Rings, Phys. Rev. D71 (2005) 025013; hep-th/0408010.

[26] J. P. Gauntlett and J. B. Gutowski, General Concentric Black Rings, Phys. Rev. D71 (2005) 045002; hep-th/0408122. 